University of Wollongong

Research Online

Faculty of Engineering and Information

Faculty of Engineering and Information

Sciences - Papers: Part B

Sciences

2019

Rapid, one-step preparation of SERS substrate in microfluidic channel for detection of molecules and heavy metal ions

Sheng Yan

Chongqing University, The Hong Kong University of Science And Technology, shengy@uow.edu.au

Fangjia Chu

Chongqing University

Haiyun Zhang

Chongqing University

Yuan Yuan

Chongqing University

Yingzhou Huang

Chongqing University

See next page for additional authors

Follow this and additional works at: https://ro.uow.edu.au/eispapers1

Part of the Engineering Commons, and the Science and Technology Studies Commons

Research Online is the open access institutional repository for the University of Wollongong. For further information contact the UOW Library: research-pubs@uow.edu.au 


\title{
Rapid, one-step preparation of SERS substrate in microfluidic channel for detection of molecules and heavy metal ions
}

\author{
Abstract \\ On-chip fabrication of surface-enhanced Raman spectroscopy (SERS)-active materials enables \\ continuous, real-time sensing of targets in the microfluidic chip. However, the current techniques require \\ the time-consuming, complicated process and costly, bulky facilities. In this work, we present a novel \\ method for synthesis of Ag nanostructures in a microfluidic channel via one-step electroless galvanic \\ replacement reaction. The whole reaction could be achieved $<10>$ mins, while the traditional methods take \\ hours. The microfluidic channel has a $\mathrm{Cu}$ base, which can reduce Ag ions to Ag nanoparticles in the \\ presence of $\mathrm{AgNO}_{3}$ solution. The new technique enables the label-free sensing of chemical molecules \\ (i.e., methylene blue)and biomolecules (i.e., urea). Two proof-of-concept experiments are performed to \\ verify the utilization of the prepared SERS substrate. First, the microfluidics-assisted SERS sensor is used \\ to detect $\mathrm{Hg}$ ions in aqueous solution with high sensitivity and good selectivity. Second, the fabricated \\ SERS-active material can couple with a concentration gradient generator for continuous SERS detection. \\ This simple technique can be used in any laboratory without any bulky equipment and can realize \\ numerous lab-on-a-chip applications with the integration of other microfluidic networks.

\section{Disciplines} \\ Engineering | Science and Technology Studies

\section{Publication Details} \\ Yan, S., Chu, F., Zhang, H., Yuan, Y., Huang, Y., Liu, A., Wang, S., Li, W., Li, S. \& Wen, W. (2019). Rapid, one- \\ step preparation of SERS substrate in microfluidic channel for detection of molecules and heavy metal \\ ions. Spectrochimica Acta Part A: Molecular and Biomolecular Spectroscopy, 220 117113-1-117113-8.

\section{Authors} \\ Sheng Yan, Fangjia Chu, Haiyun Zhang, Yuan Yuan, Yingzhou Huang, Anping Liu, Shuxia Wang, Weihua Li, \\ Shunbo Li, and Weijia Wen
}




\title{
Rapid, one-step preparation of SERS substrate in microfluidic channel for detection of molecules and heavy metal ions
}

Sheng Yan ${ }^{\mathrm{a}, \mathrm{b} *}$, Fangjia Chu ${ }^{\mathrm{a}}$, Haiyan Zhang ${ }^{\mathrm{a}}$, Yuan Yuan ${ }^{\mathrm{a}}$, Yingzhou Huang,c, Anping $\mathrm{Liu}^{\mathrm{a}, \mathrm{c}}$, Shuxia Wang ${ }^{\mathrm{a}}$, Weihua $\mathrm{Li}^{\mathrm{d}}$, Shunbo Li ${ }^{\mathrm{e} *}$, and Weijia Wen ${ }^{\mathrm{a}, b^{*}}$

a Chongqing Key Laboratory of Soft Condensed Matter Physics and Smart Materials, College of Physics, Chongqing University, Chongqing, 401331, China

b Department of Physics, The Hong Kong University of Science and Technology, Clear Water Bay, Kowloon, Hong Kong, China.

c Institute of Industrial Technology, Chongqing University, Chongqing 400044, China

d School of Mechanical, Materials, Mechatronic and Biomedical Engineering, University of Wollongong, Wollongong, NSW 2522, Australia.

e Key Disciplines Laboratory of Novel Micro-Nano Devices and System Technology and Key Laboratory of Optoelectronic Technology and Systems, Ministry of Education, School of Optoelectronics Engineering, Chongqing University, Chongqing 400044, China.

(*) Corresponding author’s e-mail address: shunbo.li@cqu.edu.cn (Shunbo Li ); sy034@uowmail.edu.au (Sheng Yan); phwen@ust.hk (Weijia Wen)

\begin{abstract}
On-chip fabrication of surface-enhanced Raman spectroscopy (SERS)-active materials enables continuous, real-time sensing of targets in the microfluidic chip. However, the current techniques require the time-consuming, complicated process and costly, bulky facilities. In this work, we present a novel method for synthesis of Ag nanostructures in a microfluidic channel via one-step electroless galvanic replacement reaction. The whole reaction could be achieved in less than 10 mins, while the traditional methods take hours. The microfluidic channel has a $\mathrm{Cu}$ base, which can reduce $\mathrm{Ag}$ ions to $\mathrm{Ag}$ nanoparticles in the presence of $\mathrm{AgNO}_{3}$ solution. The new technique enables the label-free sensing of chemical molecules (i.e., methylene blue) and biomolecules (i.e., urea). Two proof-of-concept experiments are performed to verify the utilization of the prepared SERS substrate. First, the microfluidics-assisted SERS sensor is used to detect Hg ions in aqueous solution with high sensitivity and good selectivity. Second, the fabricated SERS-active material can couple with a concentration gradient generator for continuous SERS detection. This simple technique can be used in any laboratory without any bulky equipment and can realize numerous lab-on-a-chip applications with the integration of other microfluidic networks.
\end{abstract}

Keywords: Microfluidics; Surface-enhanced Raman spectroscopy; Nanoparticle synthesis; Biosensor; Heavy metal detection

\section{Introduction}

Raman spectroscopy provides the fingerprint spectra that enable label-free sensing of molecules [1-2]. However, the overall intensities of Raman scattering signals are either too weak or too hard to realize the multiplexed sensing due to the low spectral resolution [3]. There is a demand to develop in-situ techniques that can detect 
molecules with ultrahigh sensitivity and high spectral resolution. Surface-enhanced Raman spectroscopy (SERS) [4-5] is one such technique with high sensitivity to detect targets on the single-molecule level on the surface with noble metal (e.g., Au, $\mathrm{Ag}$ and $\mathrm{Cu}$ ) nanostructures [6-7]. SERS spectra of molecules are enhanced $10^{10}$ to $10^{11}$ times over Raman signals [8]. The SERS enhancement is attributed to localized surface plasmon resonances (LSPR) of noble metal nanostructures [9-10]. SERS-based detection has been widely used in numerous research fields including environmental science [11], medicine [12-14] and cell biology [15-16].

Continuous SERS detection can be realized when SERS is coupled with a microfluidic channel [17-19]. To obtain the SERS-integrated microfluidic platform, the SERS active materials should be fabricated inside the microfluidic channel. Several deposition methods have been developed to fabricate the SERS-active metallic nanostructures, such as E-beam evaporator [20-21], laser deposition [22-23], Langmuir-Blodgett techniques [24], and thermal evaporation [25]. However, the deposition methods should be conducted under high vacuum and high temperature conditions using bulky, expensive facilities [26]. To generate the metallic nanostructures, lithography or post-treatment (e.g., annealing [25] and plasma treatment [27]) is involved, which also makes the fabrication complicated.

On-chip fabrication of SERS-active materials is a facile approach that integrates the nanostructure formation and SERS detection without lithography or annealing. One of such techniques is laser direct writing [28], which fabricates the Ag SERS substrates via photo-reduction of silver ions. The Ag substrates could be precisely located by controlling the focused laser point. However, the laser direct writing requires femtosecond laser source and extra optical elements for laser focusing. The polyol reduction reaction is another method, enabling the on-chip fabrication of SERS materials [27, 29]. The polyols can act as the solvent and the reducing agent for the metal ions [30]. The process is normally carried out at high temperatures and the involvement of polyols might bring the disturbance during SERS measurement.

Here, we report a method that can synthesize Ag nanostructures in the microfluidic channel via electroless galvanic displacement reaction between Ag and $\mathrm{Cu}$. To our best knowledge, the reported methods [21, 23] to fabricate SERS substrate using galvanic displacement reaction always involve bulky equipment (e.g., e-beam evaporator and femtosecond laser micro-machining system) and complex procedures (e.g., electrodeposition, wet etching and annealing). However, the proposed method for fabrication of SERS substrate does not require complicated fabrication process and costly facilities, and can be achieved at room temperature and atmospheric pressure. No contamination is involved during the fabrication due to the absence of polyols. The microfluidic channel can not only assist the fabrication of metallic nanostructure, but also form continuous flow for SERS detection. The capability of this method to detect solutions containing methylene blue (MB) and urea is verified. Furthermore, we demonstrate that the microfluidics-assisted SERS sensor can be applied for monitoring the water pollution by detection of $\mathrm{Hg}$ ions. With the integration of a concentration gradient generator, the fabricated SERS substrate can realize label-free SERS detection. 


\section{Materials and methods}

\subsection{Chemicals and reagents}

The materials for fabrication of metallic nanostructures include $\mathrm{AgNO}_{3}$ (Aladdin), ethanol (Aladdin), copper tape (3J), $\mathrm{H}_{2} \mathrm{SO}_{4}$ (Aladdin) and $\mathrm{HAuCl}_{4}$ (Aladdin). The molecules used for the SERS measurement include Methylene blue (Aladdin) and urea (Aladdin). The chemicals for sensing selectivity of Hg (II) against other metal species include $\mathrm{HgCl}_{2}$ (Aladdin), $\mathrm{NaCl}$ (Aladdin), $\mathrm{CaCl}_{2}$ (Aladdin), $\mathrm{CuCl}_{2}$ (Aladdin), $\mathrm{BaCl}_{2}$ (Aladdin), and $\mathrm{CdCl}_{2}$ (Aladdin). Except for $\mathrm{HgCl}_{2}$, the other metal salts have the same concentration of $1 \times 10^{-4} \mathrm{M}$. $\mathrm{NaBH}_{4}$ (Aladdin) is added to all the metal ion solutions in the selectivity test.

\subsection{Instrumentation}

Energy dispersive X-ray spectroscopy (EDS) was performed with a FE-SEM system (JSM-7800F, Joel). Scanning electron microscopy (SEM) images were taken to view the surface morphologies of the Ag nanostructures by using a scanning electron microscope (Mira 3 FE-SEM, Tescan).

\subsection{Fabrication of microfluidics-assisted SERS sensor}

The development of microfluidics-assisted SERS sensor involves two main steps: (i) bonding PDMS channel onto the copper tape, and (ii) forming Ag thin film via galvanic replacement reaction at room temperature (Fig. 1a). The SU-8 mold for microfluidic channels was fabricated using conventional photolithography (SU-8 2050, MicroChem, $50 \mu \mathrm{m}$ thickness) [31-33]. A PDMS mixture (Sylgard 184, Dow Corning) was prepared and casted on the SU-8 mold to form PDMS channel (Fig. 1b). The microfluidic channel was $200 \mu \mathrm{m}$ wide and $14 \mathrm{~mm}$ long. Next, the inlet and outlet were punched on the PDMS slab via 22G needle. The thickness of PDMS is about $2 \mathrm{~mm}$. The PDMS channel and copper tape attached on a glass substrate were treated with oxygen plasma for permanent bonding (Fig. 1c). Though the bonding strength between PDMS and copper is not stronger than the conventional siloxane bond [34] between PDMS and glass, we did not observe the leakage with flow rate as high as $50 \mu \mathrm{L} \mathrm{min}{ }^{-1}$, which is sufficient to generate steady flow in microfluidic channel for SERS substrate fabrication and detection. Then, $2 \% \mathrm{H}_{2} \mathrm{SO}_{4}$ solution was introduced into the microfluidic channel to remove the oxidation of copper tape at a flow rate of $20 \mu \mathrm{L} \mathrm{min}^{-1}$ via a syringe pump (LSP02-1B, Longer Pump), followed by introducing the $2 \mathrm{mM} \mathrm{AgNO}$ solution with the flow rate of $10 \mu \mathrm{L} \mathrm{min}{ }^{-1}$. Since the standard electrochemical potential of $\mathrm{Cu}(0.34 \mathrm{~V})$ is lower than that of $\mathrm{Ag}(0.80 \mathrm{~V})$ [35], $\mathrm{Ag}^{+}$could be reduced to elemental $\mathrm{Ag}$ by receiving electrons from copper tape when the $\mathrm{AgNO}_{3}$ solution was injected into the microfluidic channel with $\mathrm{Cu}$ base. The $\mathrm{Ag}$ nuclei formed on the $\mathrm{Cu}$ surface and then grow into $\mathrm{Ag}$ nanoparticles under the continuous flow of $\mathrm{AgNO}_{3}$ solution. Due to the spatial confinement of the microfluidic channel, Ag nanostructures only forms on the bottom of the channel, that is, on the surface of $\mathrm{Cu}$ substrate within the microfluidic channel (Fig. 1d). The obtained Ag film was then cleaned with ethanol at a flow rate of $40 \mu \mathrm{L} \mathrm{min}{ }^{-1}$. Further, the target molecules could be introduced for SERS detection. As for the acquisition of 
SERS signals, a helium-neon laser (632.8 nm, Melles Griot) and a spectrometer (iHR550, Horiba, equipped with a charge-coupled device camera) were integrated to an inverted microscope (IX73, Olympus). The excitation and collection of light were done by a $50 \times$ objective lens (NA 0.5 ). The proposed method for fabrication of SERS substrate within a microfluidic channel is simple and low cost, which avoids the high temperature particle synthesis and complicated fabrication processes. Furthermore, the SERS material (Ag nanostructures) can be newly produced prior to each experiment due to the ease of fabrication, which circumvents the adverse effect of the oxidation of Ag nanostructures. Our proposed method is not limited to the on-chip fabrication of $\mathrm{Ag}$ nanostructures. By introducing the $\mathrm{HAuCl}_{4}$ solution into the microfluidic channel, the Au nanostructures can be produced for SERS sensing (Fig. S1).

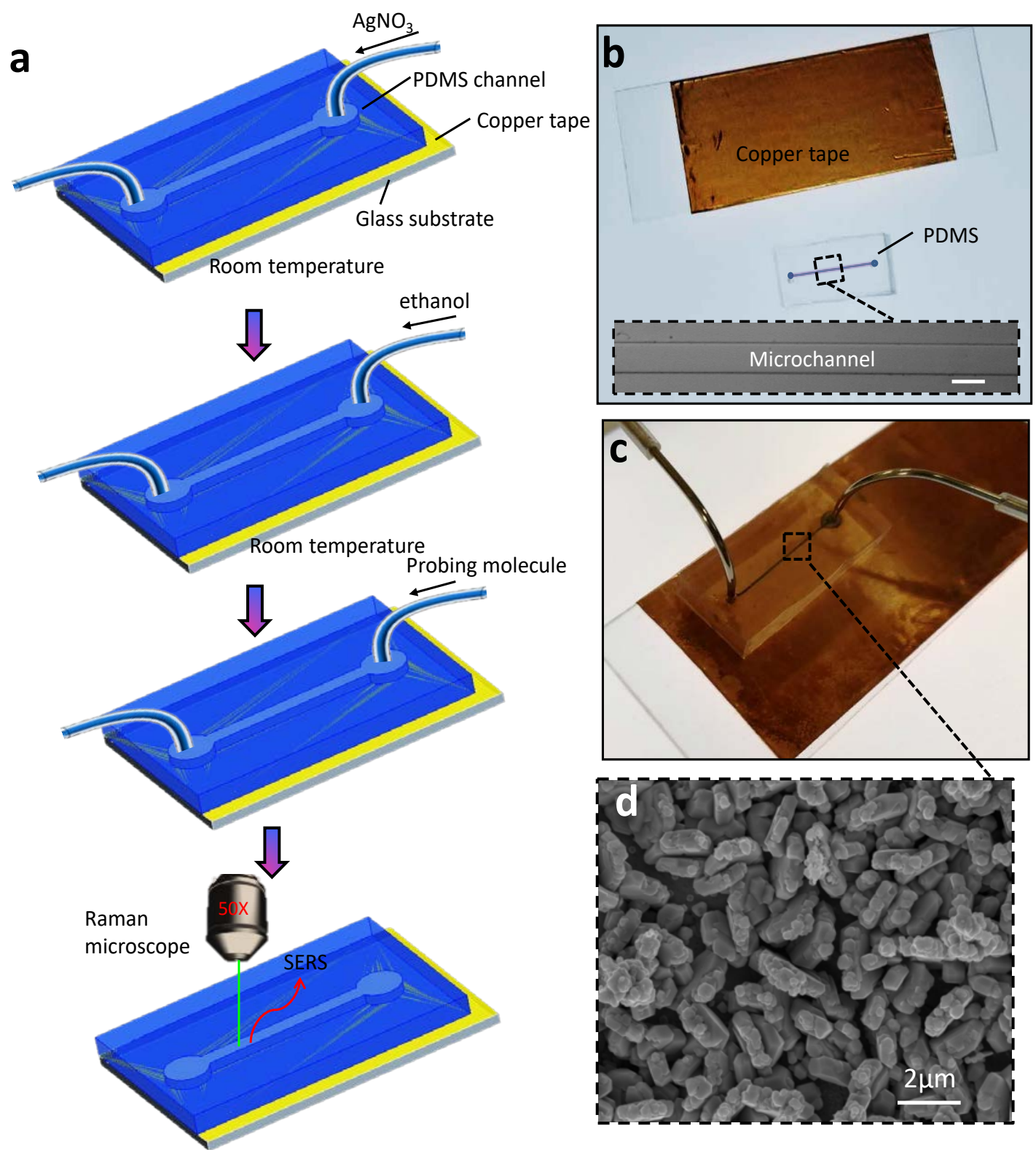

Figure 1 (a) A schematic of microfluidics-assisted SERS sensor. The Ag nanostructures were 
obtained inside the microfluidic channel via electroless galvanic replacement reaction. After washing by the ethanol, the probing molecules were introduced for SERS detection. (b) A photograph showing the components of microfluidics-assisted SERS sensor, consisting of a PDMS channel and $\mathrm{Cu}$ base. The inset is the optical graph of the microfluidic channel. The scale bar is $200 \mu \mathrm{m}$. (c) A photograph of the assembled device. (d) SEM image of fabricated Ag nanostructures in a channel.

\section{Results and discussions}

\subsection{Ag nanostructures along the microfluidic channel for SERS detection}

In the microfluidic channel, the formation of Ag nanostructures commences when the $\mathrm{AgNO}_{3}$ solution contacts the $\mathrm{Cu}$ surface at the inlet. Therefore, the concentration of $\mathrm{Ag}^{+}$reduced along the channel length due to the reduction of $\mathrm{Ag}^{+}$. Interestingly, due to the concentration gradient of $\mathrm{Ag}^{+}$along the channel, the $\mathrm{Ag}$ nanoparticles with different physical dimensions were synthesized in the same channel. The changed morphology of Ag nanostructures along the channel will, in turn, affect the SERS signals of a target molecule. The SERS phenomenon of a target molecule originates from an intense local electromagnetic field of Ag nanostructures [27]. First, we investigated the Raman intensities along the channel. Two microfluidic SERS channels were prepared (Fig. 2a and 2b). For channel I, AgNO3 solution was injected into the channel with the single directional flow (inlet $\rightarrow$ outlet). The injection time of $\mathrm{AgNO}_{3}$ solution was 4 mins. For channel II, $\mathrm{AgNO}_{3}$ solution was injected into the channel with the bidirectional flow (inlet $\rightarrow$ outlet, 2 mins, outlet $\rightarrow$ inlet, 2 mins). After washing, the target molecule, methylene blue $\left(\mathrm{MB}, 1 \times 10^{-5} \mathrm{M}\right)$, was introduced into the microfluidic channel for SERS measurements. The SERS signals were obtained at 8 points with the interval of $2 \mathrm{~mm}$ from the inlet to outlet of the channel. Six measurements were acquired to calculate the average intensity and deviation at each point. The $1618 \mathrm{~cm}^{-1}$ peak of MB was used for analysis. The Raman scattering of PDMS will not affect the SERS measurement because it is inherently weak compared to the SERS signals of molecules (Fig. S2).

As for the channel I, the Raman intensity was the highest at the point $2 \mathrm{~mm}$ from the inlet and then decreased along the channel. The Raman intensity at the outlet was 5 times lower than that at the highest intensity region ( $2 \mathrm{~mm}$ from inlet). Since the SERS intensity decreases dramatically with the increase of the particle gap [36], the poor SERS performance at the outlet attributes to the larger particle gaps. The lower $\mathrm{Ag}^{+}$concentration at the outlet will lead to the smaller Ag nanoparticles and larger particle gaps, as evidenced by scanning electron microscope (SEM) images in Fig. 2c. At $2 \mathrm{~mm}$, the smallest particle gap was observed from SEM images, which was verified by the highest Raman intensity. Although the highest $\mathrm{Ag}^{+}$concentration occured at the inlet $(0 \mathrm{~mm})$, the dense and irregular nanostructures with large holes led to relatively low Raman intensity. As for channel II, the Raman intensity was also highest in this case at the point of $2 \mathrm{~mm}$ from the inlet. However, another peak occured at the point of $12 \mathrm{~mm}$ with the bidirectional flows. When the $\mathrm{AgNO}_{3}$ solution was perfused from the outlet, the fresh $\mathrm{Ag}^{+}$would be reduced to $\mathrm{Ag}$ nanoparticles at the outlet region, causing the improved SERS intensity. With the bidirectional 
perfusion, we can always obtain the high SERS intensity at the point of $2 \mathrm{~mm}$ from the perfusion ports. Thus, the optimal working region for effective SERS detection is at the point of $2 \mathrm{~mm}$ from the perfusion port.

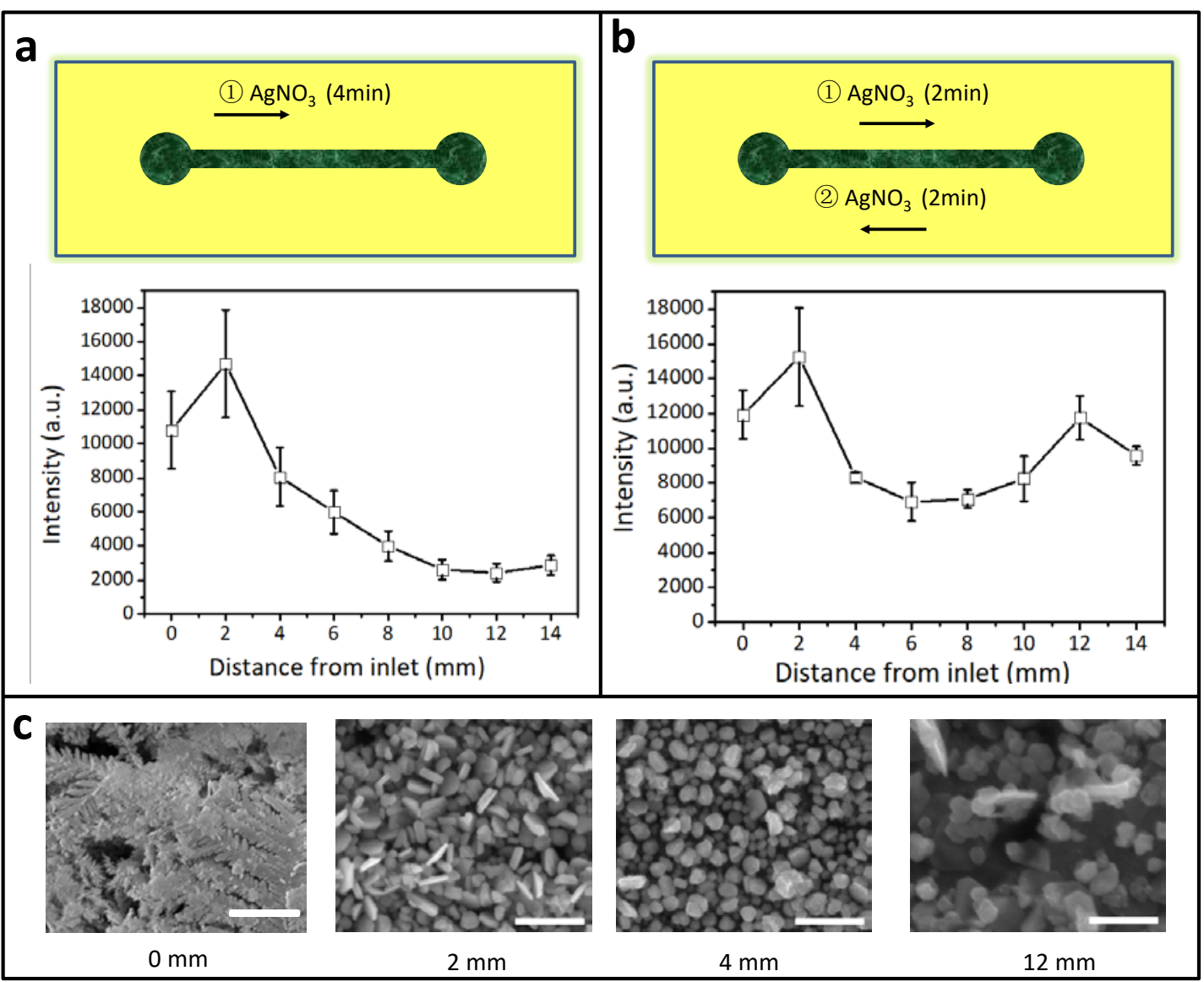

Figure 2 (a) The variations in SERS intensity of MB at $1618 \mathrm{~cm}^{-1}$ versus the distances from the inlet port. The $\mathrm{AgNO}_{3}$ solution was introduced into the channel from inlet port for 4 mins. (b) The variation in SERS intensity of MB at $1618 \mathrm{~cm}^{-1}$ versus the distance from the inlet port. The $\mathrm{AgNO}_{3}$ solution was injected into the channel with the bidirectional flow (inlet $\rightarrow$ outlet, 2 mins, outlet $\rightarrow$ inlet, 2mm). (c) SEM images showing the morphologies of Ag nanostructures along the microfluidic channel. The scale bar is $500 \mathrm{~nm}$.

\subsection{SERS detection of MB with various concentrations}

To further investigate the sensing capability of microfluidics-assisted SERS sensor, we tested the devices using $\mathrm{MB}$ solutions with different concentrations, ranging from $1 \times 10^{-8} \mathrm{M}$ to $1 \times 10^{-4} \mathrm{M}$. The SERS measurements were performed at the point of $2 \mathrm{~mm}$ from the inlet port to obtain the highest Raman intensities. Fig. 3a showed the SERS signals of MB with 5 different concentrations. The characteristic peaks of MB were apparently identified in all spectra, and the peak intensities reduced with the decrease of $\mathrm{MB}$ concentration. Once the $\mathrm{MB}$ solution was diluted to $1 \times 10^{-8}$ $\mathrm{M}$, all of the characteristic peaks of MB were lost. Therefore, the limit of detection (LOD) of this microfluidics-assisted SERS sensor was $1 \times 10^{-7} \mathrm{M}$. To verify the obtained signals were SERS phenomenon rather than normal Raman scattering, 
another microfluidic channel without the process of the galvanic reaction was prepared. Without the Ag nanostructures, the Raman scattering signal could hardly be observed at the concentration of $1 \times 10^{-5} \mathrm{M}$ (Fig. 3b), which demonstrates that all the peaks came from SERS. The enhancement factor (EF) is a key parameter to evaluate the amplification of SERS devices. The definition equation for enhancement factor (EF) applied to the case of in-channel SERS is described as [21]: EF= (ISERS/CSERS)/(INR/CNR). Where ISERs represents the intensity of the SERS signal; $I_{\mathrm{NR}}$ is the intensity of the Raman signal on a silicon substrate; CSERS and $C_{N R}$ are the concentration of molecules contributing to $I_{\mathrm{SERS}}$ and $I_{\mathrm{NR}}$, respectively. Using the SERS intensity at $1618 \mathrm{~cm}^{-1}$ for $1 \times 10^{-5} \mathrm{M} \mathrm{MB}$ in Fig. 3a and the corresponding intensity of $0.39 \mathrm{M} \mathrm{MB}$ on a non-enhanced Raman substrate (e.g., $\mathrm{Si}$ ), the calculated EF is about $1 \times 10^{6}$.

Most of the current SERS techniques require the evaporation of solute prior to the SERS measurements [37-38]. The dried analytes adhere to the SERS substrates and are hard to be washed away, which limits the repeatability of SERS substrates. However, the microfluidics assisted SERS platform can be repeatedly used to detect the target molecules adhered to the SERS substrate by physical adsorption. Since the MB can be attached to the SERS substrate via physical adsorption, the adsorbed MB can be cleaned by ethanol. As shown in Fig. 3c, the first SERS measurement was MB with the concentration of $1 \times 10^{-5} \mathrm{M}$. After on-chip washing by the ethanol for $10 \mathrm{~min}$, no SERS signal was observed at the second measurement. Then, MB with the concentration of $1 \times 10^{-6} \mathrm{M}$ was introduced into the microfluidic channel for the third SERS measurement. The obtained SERS signal of MB was disappeared again after washing, which validated that our microfluidics assisted SERS sensor can be repeatedly used for SERS detection of molecules on SERS substrate via physical adsorption.

a

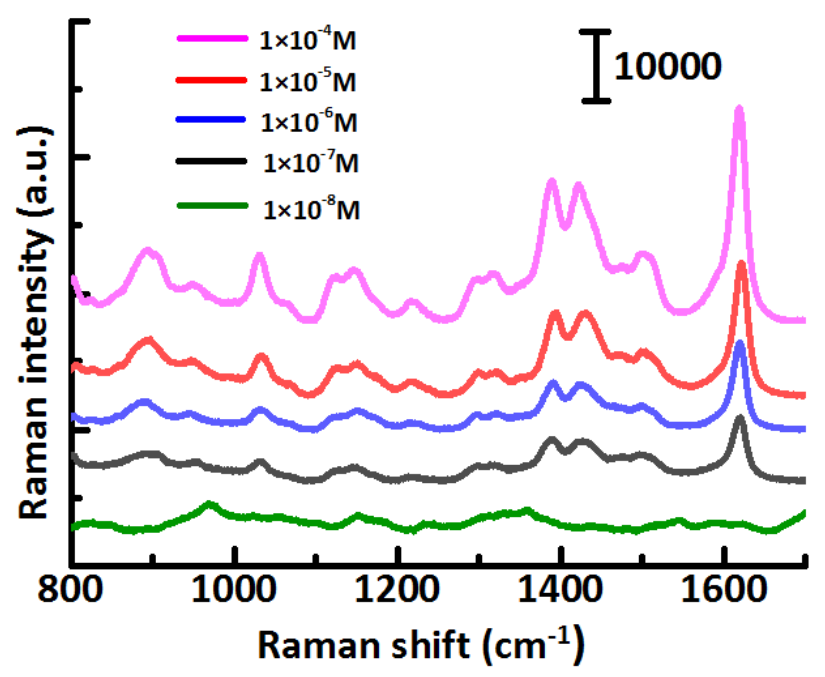

b
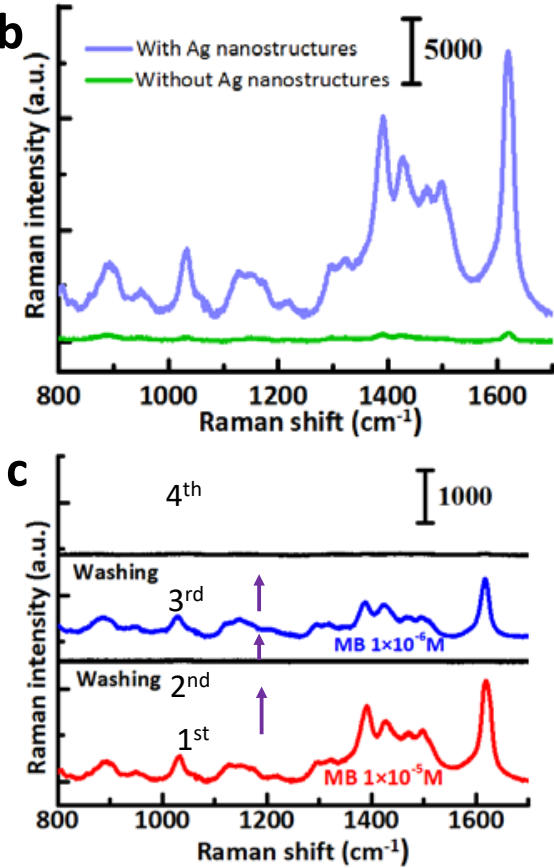

Figure 3 (a) Concentration-dependent SERS spectra of MB measured in the microfluidics-assisted 
SERS sensor. The concentration ranges from $1 \times 10^{-8} \mathrm{M}$ to $1 \times 10^{-4} \mathrm{M}$. (b) Spectra comparison of $\mathrm{MB}$ at the concentration of $1 \times 10^{-5} \mathrm{M}$ in a channel with $\mathrm{Ag}$ nanostructures and a channel without Ag nanostructures. (c) SERS spectra showing the reproducibility of the microfluidics-assisted SERS sensor.

\subsection{SERS detection of biomolecules}

To demonstrate the biocompatibility of microfluidics assisted SERS sensor, a biomolecule (i.e., urea) was utilized as target to characterize the device. Urea, a metabolite, mainly exists in human blood and urine. The detection of urea is of great significance in monitoring of kidney function. Here, urea solution was prepared at the concentrations of $1 \times 10^{-5} \mathrm{M}, 1 \times 10^{-4} \mathrm{M}, 5 \times 10^{-4} \mathrm{M}, 1 \times 10^{-3} \mathrm{M}$, and $1 \times 10^{-2} \mathrm{M}$ and then injected into the microfluidic channel using a syringe pump. The SERS measurements were obtained at the point of $2 \mathrm{~mm}$ from the inlet. After each concentration measurement, the channel was rinsed with ethanol to remove the residue molecule. Fig. 4 presented SERS spectra of urea solutions in the microfluidics-assisted SERS sensor. A characteristic peak of $1000 \mathrm{~cm}^{-1}$ was observed, which corresponded to the symmetrical C-N stretching vibration mode [39]. As previously reported SERS sensors of urea detection, the LOD of Au-coated nano-dome arrays was $11.9 \mathrm{mM}$ [40] and the LOD of nanoporous gold disk arrays was $0.67 \mathrm{mM}$ [41]. Our device with the LOD of $0.1 \mathrm{mM}$ has higher sensitivity for clinical applications such as kidney function monitoring and urine analysis.

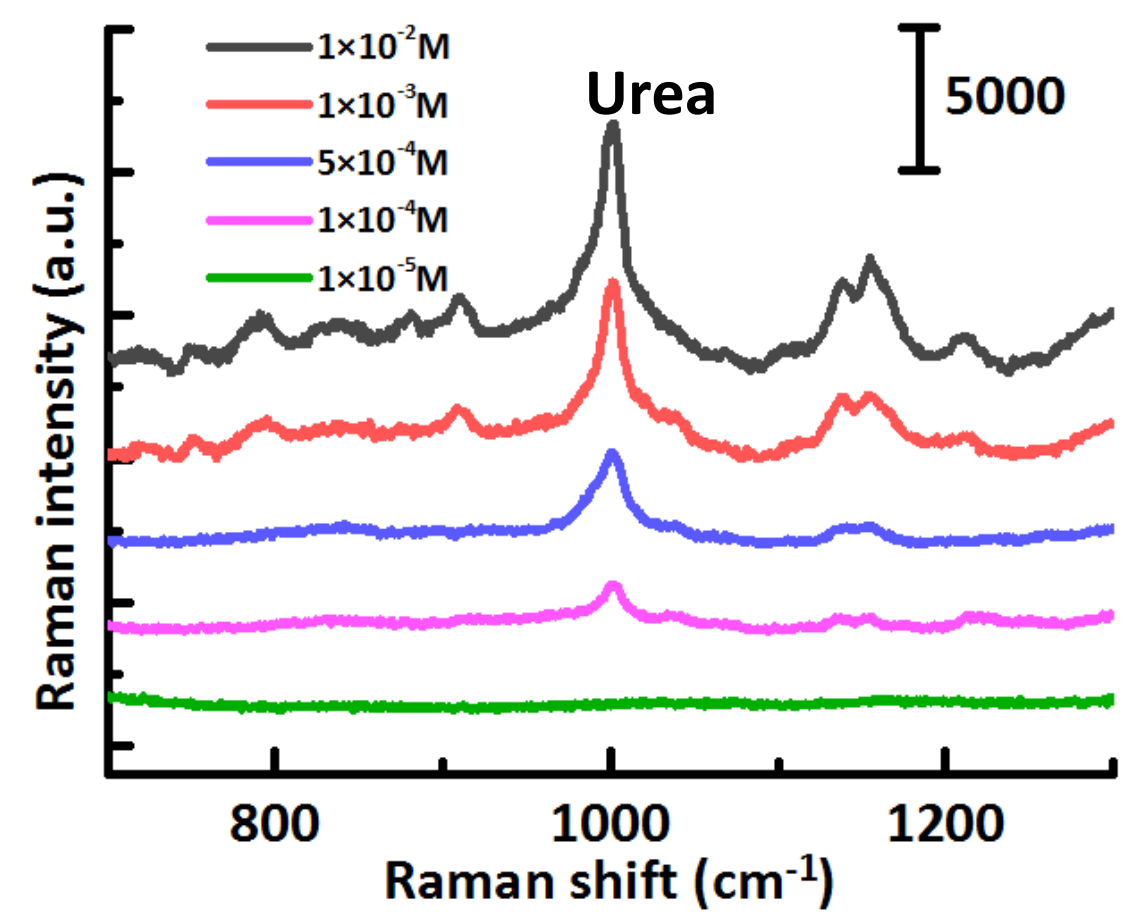

Figure 4 Concentration dependent SERS spectra of urea measured in the microfluidics-assisted SERS sensor. The concentration ranges from $1 \times 10^{-5} \mathrm{M}$ to $1 \times 10^{-2} \mathrm{M}$.

\subsection{Mercury (II) detection}

Except for the application in the detection of biomolecules, the microfluidics 
assisted SERS sensor can also be utilized to detect the Hg (II) for environmental monitoring and evaluation of wastewater treatment. Hg (II) is toxic to human beings and animals, even at low concentrations. Currently, there are two SERS-based methods for detection of $\mathrm{Hg}$ (II) in solutions. The first one is that $\mathrm{Hg}$ (II) links a Raman probe (e.g., DNA enzyme [42] or $\mathrm{T}-\mathrm{Hg}^{2+}-\mathrm{T}$ pairs [43]). With the increase of $\mathrm{Hg}$ (II) concentration, the SERS intensities of marker molecules also increase, which can detect the Hg (II). The second method is based on the amalgamation of $\mathrm{Hg}$ (II) with the SERS active metal nanoparticles [44]. With the reduction of $\mathrm{Hg}$ (II) into $\mathrm{Hg}$ (0) on the surface of nanoparticles to form Hg/Ag shell (Fig. 5a), the SERS intensities associated with the Raman marker molecules will decrease. Based on this phenomenon, the Hg (II) could be detected in the microfluidics assisted SERS sensor with Ag nanostructures.

The microfluidics assisted SERS sensor was prepared using the same method described before. The Hg (II) solutions with various concentrations $\left(1 \times 10^{-9} \mathrm{M}\right.$ to $1 \times 10^{-3} \mathrm{M}$ ) were separately introduced into the channel for $10 \mathrm{~min}$. The addition of sodium borohydride $\left(\mathrm{NaBH}_{4}\right)$ in $\mathrm{Hg}$ (II) solutions could accelerate the amalgamation process [45]. The addition was conducted just prior to injection into microfluidic chip in the current experiment. It is noted that $\mathrm{SnCl}_{2}$ is an alternative for $\mathrm{Hg}$ (II) reduction, but with slow reaction speed compared to $\mathrm{NaBH}_{4}$. The $\mathrm{Hg}$ amalgamation was further verified by the energy dispersive X-ray spectroscopy (EDS), as evidenced by Fig. 5b. The substrate consisted of $\mathrm{Cu}$ (copper base), Ag and Hg. Thus, Hg was reduced on the Ag nanostructures. Then, the $\mathrm{MB}$ at the concentration of $1 \times 10^{-5} \mathrm{M}$ was pumped into the channel, acting as a Raman marker. The $1618 \mathrm{~cm}^{-1}$ peak of MB collected in different concentrations of $\mathrm{Hg}$ (II) was analyzed to evaluate the sensitivity of our device in detecting $\mathrm{Hg}$ (II). The relation between the concentration of $\mathrm{Hg}$ (II) and the corresponding SERS intensities was plotted in Fig. 5c. Three phases were observed in the change of SERS intensity with the increasing concentration of $\mathrm{Hg}$ (II). In the first phase, Hg (II) at very low concentration $\left(<1 \times 10^{-8} \mathrm{M}\right)$ did not affect the SERS intensities of Raman markers due to the weak interaction between $\mathrm{Hg}$ (II) and $\mathrm{Ag}$ nanostructures. In the second phase, when the $\mathrm{Hg}$ (II) was higher than $1 \times 10^{-7} \mathrm{M}$, a dramatic decrease in the SERS intensity with the increase of $\mathrm{Hg}$ (II) concentration was observed. This was attributed to the formation of $\mathrm{Hg} / \mathrm{Ag}$ shell on the $\mathrm{Ag}$ nanostructures, which influenced the LSPR of Ag nanostructures. In the last phase, almost no SERS signal was observed. The LOD of our device for detection of $\mathrm{Hg}$ (II) is $1 \times 10^{-7} \mathrm{M}$ because the SERS intensity begins to decrease when the $\mathrm{Hg}$ (II) in aqueous solution is over $1 \times 10^{-7} \mathrm{M}$. To further lower the detection limit required by Environmental Protection Agency (10 nM), colloidal lithography may be used to obtain the more uniform metallic nanostructures [46-48]. The proposed method is a simple and fast way to prepare the SERS substrate integrated in microfluidic chip. It can be used to roughly detect the heavy pollution of mercury. Each experiment was repeated three times. The results can be steadily repeated with the deviation less than $20 \%$. To improve the reproducibility of the heavy metal ion detection, a new microfluidic chip with two inlets and mixing structures could be designed to conduct the in-situ reduction reaction of $\mathrm{Hg}$ (II) in the microfluidic channel. 

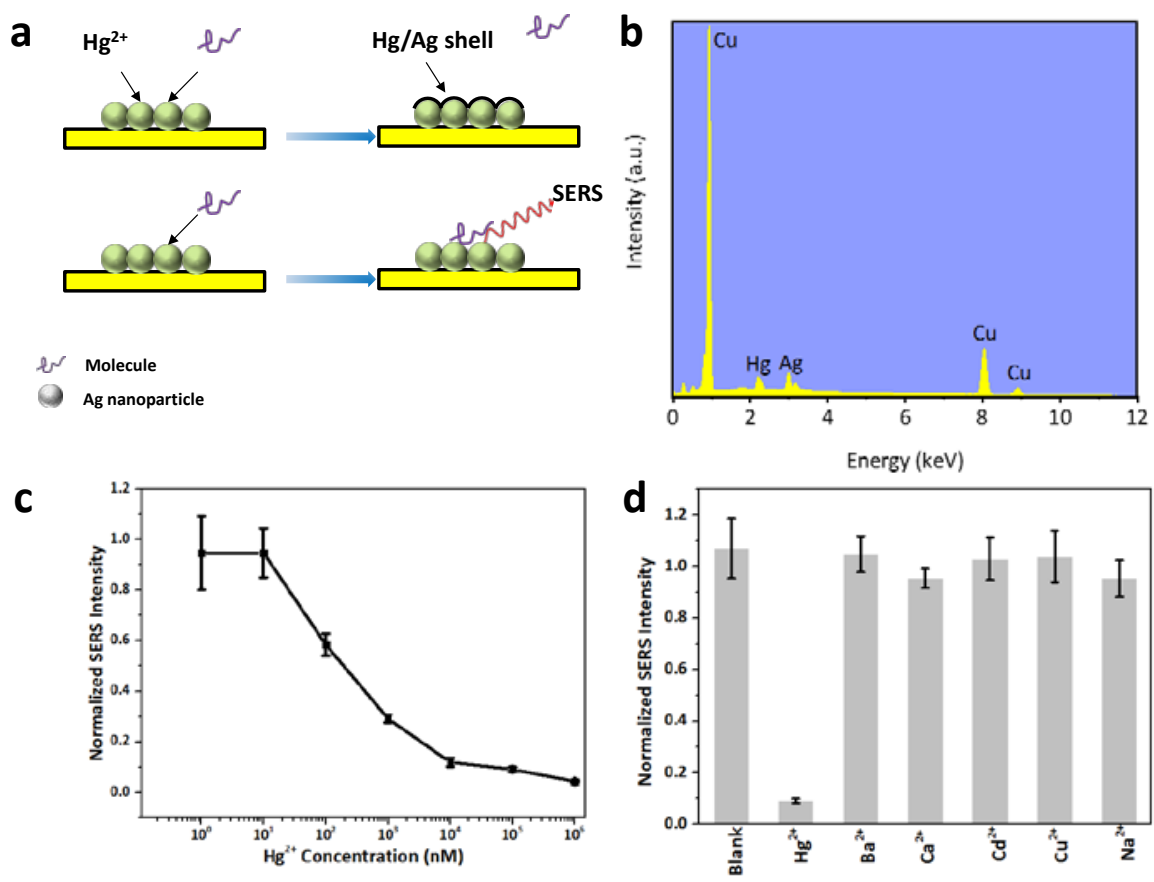

Figure 5 (a) Schematic description of the detection of $\mathrm{Hg}$ (II) on the Ag particles. (b) EDS data of SERS-active substrate. The substrate consists of $\mathrm{Cu}$ (base), Hg, and Ag. (c) The normalized SERS intensity of the peak at $1618 \mathrm{~cm}^{-1}$ versus the $\mathrm{Hg}$ (II) concentration. (d) The selectivity of the proposed SERS detection Hg (II) against other metal ions. Each measurement was repeated three times.

Our microfluidics-assisted SERS sensor also has good selectivity in the detection of Hg (II). As shown in Fig. 5d, five divalent metal ions are used and none of them can reduce the SERS intensity as much as that induced by $\mathrm{Hg}$ (II), demonstrating that the proposed method has great potential to detect the contamination of $\mathrm{Hg}$ (II) in aquatic ecosystems. It has to be noted that the reduction of heavy metal ions (e.g $\mathrm{Cu}$ and $\mathrm{Cd}$ ) happened in the reactions. However, they had no amalgamation effect as Hg did. Therefore, the proposed method had good selectivity in detection of $\mathrm{Hg}$ ions.

\subsection{SERS detection in the concentration gradient generator}



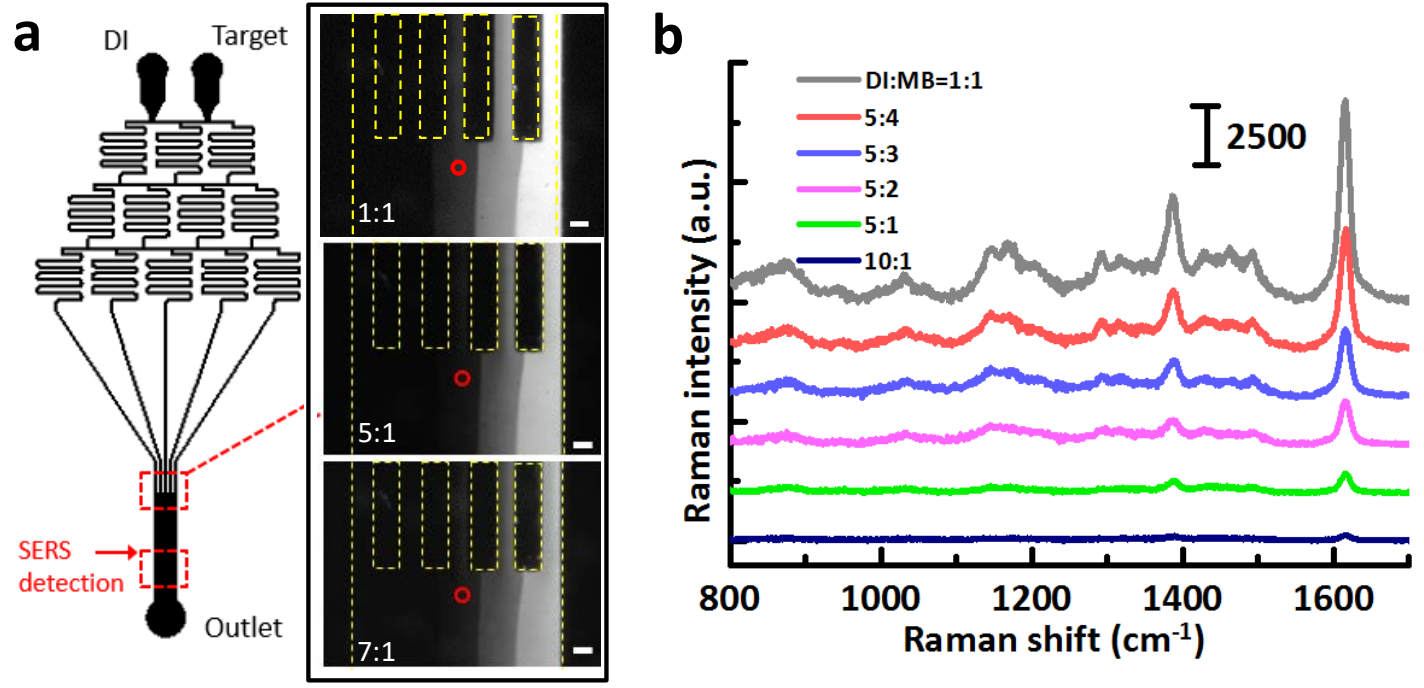

Figure 6 (a) SERS detection of molecules in the concentration gradient generator. DI water and the target molecules can spatially generate the concentration gradient through the "Christmas tree" channel as shown in the fluorescent images. By varying the flow rate ratio, the concentration at the same location can be changed. (b) The SERS intensity of MB molecules gradually increases with the decrease of the flow rate ratio of DI water and MB solution.

The microfluidics assisted SERS sensor could achieve more complex functions by integrating with an advanced microfluidic channel. Here, a "Christmas tree" channel was designed, which could generate the spatial concentration gradient by fluidic mixing [49], as shown in Fig. 6a. The channel had two inlets and one outlet. In this experiment, the $\mathrm{AgNO}_{3}$ solution was introduced into the channel via outlet port to form the Ag nanostructures, as described above. The DI water and the target molecule - MB as a test sample were injected through the left side inlet and right side inlet, respectively. The flow rate of DI water was set to be constant $\left(10 \mu \mathrm{L} \mathrm{min}{ }^{-1}\right)$. By varying the flow rate of the target molecule, the concentration gradient can be dynamically changed. To visualize the concentration gradient in the channel, the fluorescent molecule, Rhodamine $\mathrm{B}$, was pumped into the channel. The images were taken under the fluorescent field (Fig. 6a). The two inlets were divided into five channels through a three-level structure. The concentration gradient was successfully formed after the fluids passed mixing units and then merged into a single channel. The fluorescent intensity at the red circle was decreased when the flow rate of Rhodamine

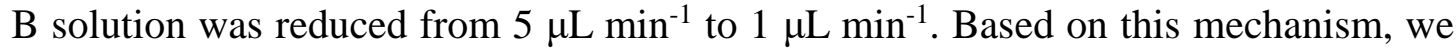
could detect the non-fluorescent molecules with the integration of SERS. The MB at the concentration of $1 \times 10^{-5} \mathrm{M}$ was pumped into the channel via the right-side inlet. The SERS measurement was also done at the same location (middle of the channel) but with a different flow rate of MB. The flow rate ratio of DI water and the MB solution was varied from 10:1 to 1:1. The SERS signal with the main peak of MB at $1618 \mathrm{~cm}^{-1}$ was clearly observed with a time delay of $20 \mathrm{~s}$ from the experiments and increased with the increase of flow rate of MB solution (Fig. 6b). This experiment demonstrates that the SERS substrate is able to detect the molecules with the 
integration of a concentration gradient generator. The good compatibility with other microfluidic networks enables the microfluidics assisted SERS sensor to achieve diverse functions.

\section{Conclusions}

This work described a simple, microfluidics-assisted method to fabricate the SERS-active materials. The silver nanostructures were prepared inside the microfluidic channel via one-step electroless galvanic replacement reaction. The various particle sizes were obtained along the microfluidic channel and the point of 2 $\mathrm{mm}$ from the inlet port was found to produce the highest Raman signal. The microfluidics-assisted SERS sensor had been demonstrated its capability to detect the chemical molecule (e.g., MB) and biomolecules (e.g., urea). The microfluidics assisted SERS sensor was further used to detect the Hg ions in the aqueous solution, which showed LOD of $1 \times 10^{-7} \mathrm{M}$ and good selectivity against other metal ions. In addition, a proof-of-concept experiment was developed to show that our method could realize SERS detection with the integration of a concentration gradient generator.

The developed microfluidics-assisted SERS sensor has several advantages. First, this platform can integrate the nanoparticle synthesis, fluidic introduction, and SERS detection in a single chip. Second, the microfluidic channel can spatially and temporally confine the fluids into the small area. Due to this unique property, the different particle size can be produced in the process of particle synthesis via electroless galvanic reaction. The molecules were introduced to the channel with the exact alignment with the SERS substrate. Third, the device can be repeatedly used for SERS detection of molecules on the SERS substrate via physical adsorption. Last, the microfluidics-assisted SERS sensor can couple with a concentration gradient generator to form a label-free sensing system. We envision that our proposed technique can achieve diverse functions for lab-on-a-chip applications by integrating with other microfluidic networks.

\section{Conflicts of interest}

There are no conflicts of interest to declare.

\section{Authors' contributions}

SY, FC, HZ and YY conducted the experiments. SY, SL, and WW contributed most of the ideas and supervised all experiments and theory. SY, SL, WL, and WW wrote the paper. $\mathrm{YH}, \mathrm{SW}, \mathrm{AL}$ conducted the material characterization. All authors discussed the results and commented on the manuscript. All authors read and approved the final manuscript.

\section{Acknowledgements}

Dr. Sheng Yan is the recipient of the 2018 Endeavour Research Fellowship funded by the Australian Department of Education and Training. Prof. Wang and Prof. Huang thank the support from the Fundamental Research Funds for the Central Universities (2018CDXYWU0025, 2018CDJDWL0011). Dr. Li would like to thank the financial support by 'Shenzhen Peacock Plan' (No. KQTD2016022614361432) and Fundamental Research Funds for the Central Universities (2018CDXYGD0017). Prof. 
Liu would like to thank the financial support by the National Natural Science Foundation of China (No. 61575033).

\section{References}

1.M. Moskovits, Surface-enhanced spectroscopy. Review of Modern Physics, 57 (1985), 783-826.

2.K. Hashimoto,M. Takahashi,T. Ideguchi and K. Goda, Broadband coherent Raman spectroscopy running at 24,000 spectra per second. Scientific reports, 6 (2016), 21036.

3.S.-Y. Ding,J. Yi,J.-F. Li,B. Ren,D.-Y. Wu,R. Panneerselvam and Z.-Q. Tian, Nanostructure-based plasmon-enhanced Raman spectroscopy for surface analysis of materials. Nature Reviews Materials, 1 (2016), 16021.

4.M. Fleischmann,P. J. Hendra and A. J. McQuillan, Raman spectra of pyridine adsorbed at a silver electrode. Chem. Phys. Lett., 26 (1974), 163-166.

5.D. L. Jeanmaire and R. P. Van Duyne, Surface Raman spectroelectrochemistry: Part I. Heterocyclic, aromatic, and aliphatic amines adsorbed on the anodized silver electrode. Journal of electroanalytical chemistry and interfacial electrochemistry, 84 (1977), 1-20.

6.S. Nie and S. R. Emory, Probing single molecules and single nanoparticles by surface-enhanced Raman scattering. Science, 275 (1997), 1102-1106.

7.K. Kneipp,Y. Wang,H. Kneipp,L. T. Perelman,I. Itzkan,R. R. Dasari and M. S. Feld, Single molecule detection using surface-enhanced Raman scattering (SERS). Phys. Rev. Lett., 78 (1997), 1667.

8.B. Sharma,R. R. Frontiera,A.-I. Henry,E. Ringe and R. P. Van Duyne, SERS: Materials, applications, and the future. Materials today, 15 (2012), 16-25.

9.V. Bastys,I. Pastoriza - Santos,B. Rodrí guez - González,R. Vaisnoras and L. M. Liz - Marzán, Formation of silver nanoprisms with surface plasmons at communication wavelengths. Adv. Funct. Mater., 16 (2006), 766-773.

10.S. Yang,W. Cai,L. Kong and Y. Lei, Surface Nanometer - Scale Patterning in Realizing Large Scale Ordered Arrays of Metallic Nanoshells with Well - Defined Structures and Controllable Properties. Adv. Funct. Mater., 20 (2010), 2527-2533.

11.R. A. Alvarez-Puebla,D. S. dos Santos Jr and R. F. Aroca, SERS detection of environmental pollutants in humic acid-gold nanoparticle composite materials. Analyst, 132 (2007), 1210-1214.

12.H. Hwang,D. Han,Y.-J. Oh,Y.-K. Cho,K.-H. Jeong and J.-K. Park, In situ dynamic measurements of the enhanced SERS signal using an optoelectrofluidic SERS platform. Lab Chip, 11 (2011), 2518-2525. 13.F. Zeng,W. Duan,B. Zhu,T. Mu,L. Zhu,J. Guo and X. Ma, Based Versatile Surface-Enhanced Raman Spectroscopy Chip with Smartphone-Based Raman Analyzer for Point-of-Care Application. Anal. Chem., 91 (2018), 1064-1070.

14.S. S. Panikar,G. Ramírez-García,S. Sidhik,T. Lopez-Luke,C. Rodriguez-Gonzalez,I. H. Ciapara,P. S. Castillo,T. Camacho-Villegas and E. De la Rosa, Ultrasensitive SERS Substrate for Label-Free Therapeutic-Drug Monitoring of Paclitaxel and Cyclophosphamide in Blood Serum. Anal. Chem., 91 (2018), 2100-2111.

15.J. Ando,K. Fujita,N. I. Smith and S. Kawata, Dynamic SERS imaging of cellular transport pathways with endocytosed gold nanoparticles. Nano Lett., 11 (2011), 5344-5348.

16.A. F. Chrimes,K. Khoshmanesh,S. Y. Tang,B. R. Wood,P. R. Stoddart,S. S. Collins,A. Mitchell and K. Kalantar-Zadeh, In situ SERS probing of nano-silver coated individual yeast cells. Biosensors \& bioelectronics, 49 (2013), 536-541. 
17.H. Mao,W. Wu,D. She,G. Sun,P. Lv and J. Xu, Microfluidic Surface - Enhanced Raman Scattering Sensors Based on Nanopillar Forests Realized by an Oxygen - Plasma - Stripping - of - Photoresist Technique. Small, 10 (2014), 127-134.

18.K. R. Strehle,D. Cialla,P. Rösch,T. Henkel,M. Köhler and J. Popp, A reproducible surface-enhanced Raman spectroscopy approach. Online SERS measurements in a segmented microfluidic system. Anal. Chem., 79 (2007), 1542-1547.

19.S. Li,W. Cao,Y. S. Hui and W. Wen, Simple and reusable picoinjector for liquid delivery via nanofluidics approach. Nanoscale research letters, 9 (2014), 147.

20.S. M. Wells,S. D. Retterer,J. M. Oran and M. J. Sepaniak, Controllable nanofabrication of aggregate-like nanoparticle substrates and evaluation for surface-enhanced Raman spectroscopy. ACS Nano, 3 (2009), 3845-3853.

21.J. Parisi,L. Su and Y. Lei, In situ synthesis of silver nanoparticle decorated vertical nanowalls in a microfluidic device for ultrasensitive in-channel SERS sensing. Lab Chip, 13 (2013), 1501-1508.

22.S. Henley,J. Carey and S. Silva, Laser-nanostructured Ag films as substrates for surface-enhanced Raman spectroscopy. Appl. Phys. Lett., 88 (2006), 081904.

23.S. Bai,D. Serien,A. Hu and K. Sugioka, 3D Microfluidic Surface - Enhanced Raman Spectroscopy (SERS) Chips Fabricated by All - Femtosecond - Laser - Processing for Real - Time Sensing of Toxic Substances. Adv. Funct. Mater., 28 (2018), 1706262.

24.Q. Zhang,Y. H. Lee,I. Y. Phang,C. K. Lee and X. Y. Ling, Hierarchical 3D SERS Substrates Fabricated by Integrating Photolithographic Microstructures and Self - Assembly of Silver Nanoparticles. Small, 10 (2014), 2703-2711.

25.S. Yang,F. Xu,S. Ostendorp,G. Wilde,H. Zhao and Y. Lei, Template - Confined Dewetting Process to Surface Nanopatterns: Fabrication, Structural Tunability, and Structure - Related Properties. Adv. Funct. Mater., 21 (2011), 2446-2455.

26.Y. Zheng,T. Thai,P. Reineck,L. Qiu,Y. Guo and U. Bach, DNA - Directed Self - Assembly of Core - Satellite Plasmonic Nanostructures: A Highly Sensitive and Reproducible Near - IR SERS Sensor. Adv. Funct. Mater., 23 (2013), 1519-1526.

27.J. Leem,H. W. Kang,S. H. Ko and H. J. Sung, Controllable Ag nanostructure patterning in a microfluidic channel for real-time SERS systems. Nanoscale, 6 (2014), 2895-2901.

28.B.-B. Xu,Z.-C. Ma,L. Wang,R. Zhang,L.-G. Niu,Z. Yang,Y.-L. Zhang,W.-H. Zheng,B. Zhao and Y. $\mathrm{Xu}$, Localized flexible integration of high-efficiency surface enhanced Raman scattering (SERS) monitors into microfluidic channels. Lab Chip, 11 (2011), 3347-3351.

29.W. Yan,L. Yang,J. Chen,Y. Wu,P. Wang and Z. Li, In Situ Two - Step Photoreduced SERS Materials for On - Chip Single - Molecule Spectroscopy with High Reproducibility. Adv. Mater., 29 (2017),

30.Y. Sun and Y. Xia, Large - scale synthesis of uniform silver nanowires through a soft, self - seeding, polyol process. Adv. Mater., 14 (2002), 833-837.

31.S. Yan,J. Zhang,G. Alici,H. Du,Y. Zhu and W. Li, Isolating plasma from blood using a dielectrophoresis-active hydrophoretic device. Lab Chip, 14 (2014), 2993-3003.

32.Li,Shunbo,Li,Ming,Li,Weihua,Hui and Cao, A novel method to construct 3D electrodes at the sidewall of;microfluidic channel. Microfluidics \& Nanofluidics, 14 (2013), 499-508.

33.S. Yan,J. Zhang,C. Pan,D. Yuan,G. Alici,H. Du,Y. Zhu and W. Li, An integrated dielectrophoresis-active hydrophoretic microchip for continuous particle filtration and separation. Journal of Micromechanics \& Microengineering, 25 (2015), 084010.

34.D. C. Duffy,J. C. McDonald,O. J. Schueller and G. M. Whitesides, Rapid prototyping of 
microfluidic systems in poly (dimethylsiloxane). Anal. Chem., 70 (1998), 4974-4984.

35.X. Xia,Y. Wang,A. Ruditskiy and Y. Xia, 25th anniversary article: galvanic replacement: a simple and versatile route to hollow nanostructures with tunable and well - controlled properties. Adv. Mater., 25 (2013), 6313-6333.

36.C. Zong,M. Xu,L.-J. Xu,T. Wei,X. Ma,X.-S. Zheng,R. Hu and B. Ren, Surface-Enhanced Raman Spectroscopy for Bioanalysis: Reliability and Challenges. Chem. Rev., (2018),

37.F. D. Angelis,F. Gentile,F. Mecarini,G. Das,M. Moretti,P. Candeloro,M. L. Coluccio,G. Cojoc,A. Accardo and C. Liberale, Breaking the diffusion limit with super-hydrophobic delivery of molecules to plasmonic nanofocusing SERS structures. Nat. Photonics, 5 (2011), 682-687.

38.W. Chen,H. Shi,F. Wan,P. Wang,Z. Gu,W. Li,L. Ke and Y. Huang, Substrate influence on the polarization dependence of SERS in crossed metal nanowires. Journal of Materials Chemistry C, 5 (2017),

39.W. R. Premasiri,R. H. Clarke and M. E. Womble, Urine analysis by laser Raman spectroscopy. Lasers Surg. Med., 28 (2001), 330-4.

40.H. Y. Wu,C. J. Choi and B. T. Cunningham, Plasmonic nanogap-enhanced Raman scattering using a resonant nanodome array. Small, 8 (2012), 2878-2885.

41.M. Li,F. Zhao,J. Zeng,J. Qi,J. Lu and W. C. Shih, Microfluidic surface-enhanced Raman scattering sensor with monolithically integrated nanoporous gold disk arrays for rapid and label-free biomolecular detection. J. Biomed. Opt., 19 (2014), 111611.

42.W. Ma,H. Yin,L. Xu,X. Wu,H. Kuang,L. Wang and C. Xu, Ultrasensitive aptamer-based SERS detection of PSAs by heterogeneous satellite nanoassemblies. Chem. Commun., 50 (2014), 9737-40.

43.F. Li,J. Wang,Y. Lai,C. Wu,S. Sun,Y. He and H. Ma, Ultrasensitive and selective detection of copper (II) and mercury (II) ions by dye-coded silver nanoparticle-based SERS probes. Biosens. Bioelectron., 39 (2013), 82.

44.W. Ren,C. Zhu and E. Wang, Enhanced sensitivity of a direct SERS technique for Hg2+ detection based on the investigation of the interaction between silver nanoparticles and mercury ions. Nanoscale, 4 (2012), 5902.

45.L. Zhang,X. Sha,Q. Fan,L. Han,Y. Yin and C. Gao, Gold nanoshurikens with uniform sharp tips for chemical sensing by the localized surface plasmon resonance. Nanoscale, 9 (2017),

46.B. Lay,Y. M. Sabri,S. J. Ippolito and S. K. Bhargava, Galvanically replaced Au-Pd nanostructures: study of their enhanced elemental mercury sorption capacity over gold. Phys. Chem. Chem. Phys., 16 (2014), 19522-19529.

47.B. Lay,A. E. Kandjani,M. H. Amin,K. M. M. Kabir,S. J. Ippolito,Y. M. Sabri and S. K. Bhargava, Galvanic replacement of colloidal monolayer crystal on a QCM device for selective detection of mercury vapor. Sensor. Actuat. B: Chem., 250 (2017), 383-392.

48.B. Lay,Y. M. Sabri,A. E. Kandjani and S. K. Bhargava, Using colloidal lithography to control the formation of gas sorption sites through galvanic replacement reaction. J. Colloid Interface Sci., 547 (2019), 199-205.

49.J. N. Li,H. Baskaran,S. K. Dertinger,G. M. Whitesides,d. W. L. Van and M. Toner, Neutrophil chemotaxis in linear and complex gradients of interleukin-8 formed in a microfabricated device. Nat. Biotechnol., 20 (2002), 826-30. 